\title{
Prevalence and characteristics of dysfunction of right ventricle in peripartum cardiomyopathy
}

This article was published in the following Dove Press journal:

Research Reports in Clinical Cardiology

Number of times this article has been viewed

\author{
Fatou Aw' \\ Mouhamadou Bamba \\ Ndiaye \\ Simon Antoine Sarr ${ }^{\prime}$ \\ Malick Bodian' \\ Aliou Alassane Ngaide ${ }^{2}$ \\ Alassane Mbaye $\mathrm{M}^{2,3}$ \\ Adama Kane' \\ Abdoul Kane \\ Maboury Diao' \\ Serigne Abdou $\mathrm{Ba}^{\prime}$ \\ 'Department of Cardiology, Aristide \\ Le Dantec Teaching Hospital, \\ ${ }^{2}$ Department of Cardiology, General \\ Hospital of Grand Yoff, ${ }^{3}$ Department \\ of Cardiology, Teaching Hospital of \\ Fann, ${ }^{4}$ Cheikh Anta Diop University of \\ Dakar, Dakar, Senegal
}

Correspondence: Fatou Aw

Department of Cardiology, Aristide Le

Dantec Teaching Hospital, PO Box 6003,

Dakar Etoile, Dakar, Senegal

Tel +22I 774220035

Fax +22133821 5521

Email fatoukineaw@yahoo.fr
Background: The study of the right ventricle (RV) is of particular interest as it is believed to be involved in several heart diseases. Peripartum cardiomyopathy (PPCM) is characterized by left ventricular systolic dysfunction occurring between the last month of pregnancy and the fifth month postpartum. It is often combined with RV dysfunction, but this has rarely been described in the literature. The main aim of this study was to assess the prevalence of RV dysfunction in a PPCM population.

Methods: We conducted a prospective and investigative study at the Cardiac Clinic of the Aristide Le Dantec Teaching Hospital in Dakar, Senegal, between March and June 2013. All patients hospitalized during the study period and meeting the diagnostic criteria for PPCM were included. Results: During the study period, 326 patients were hospitalized in the cardiology department; 19 were diagnosed with PPCM corresponding to a hospital prevalence of 5.8\%. All patients had left ventricular dysfunction, with an average ejection fraction of $23.01 \% \pm 9.73 \%$ at the Simpson biplane. Based on the tricuspid annular plane systolic excursion (TAPSE), 11 out of the 19 patients $(57.9 \%)$ had RV systolic dysfunction. Ten patients (52.6\%) had an Sa tricuspid wave (speed of the systolic wave to the tricuspid ring in tissue Doppler) below the standard. The average RV area shortening was $23.73 \% \pm 14.16 \%$, with extremes of $7.8 \%$ and $53.7 \%$. Fifteen patients $(78.9 \%)$ had a rate of shortening fraction of the surface area of RV below the standard. When the other parameters were taken into account, 15 patients $(78.9 \%)$ had at least 3 of the following indexes altered: fractional area change (FAC), Sa tricuspid, TAPSE, and the ratio between the sum of both isovolumic times and the ejection time (TEI index).

Conclusion: RV dysfunction is common in PPCM. In view of the prognostic interest on the right ventricle; its evaluation must take into account all of the measurement parameters in order to allow for better detection.

Keywords: peripartum cardiomyopathy, right ventricular dysfunction, heart failure, Dakar, Sénégal

\section{Introduction}

Peripartum cardiomyopathy (PPCM) is characterized by left ventricular (LV) systolic dysfunction (LVSD) occurring between the last month of pregnancy and the fifth month postpartum in patients without preexisting cardiac disease. ${ }^{1}$ This cardiopathy is frequent in our region and is the bed of several complications, especially thromboembolic and rhythmic complications. ${ }^{2}$ Several studies have been carried out on this population, focusing on the epidemiological, clinical, electrocardiographic and echocardiographic aspects of the left ventricle..$^{2-5}$ The real incidence of PPCM is unknown and varies widely worldwide, with a remarkable North-South Hemisphere gap, ranging from approxi- 
mately 1 per 300 live births in Haiti, 1 per 1000 live births in South Africa and up to 1 per 3000 live births in the USA and Western Europe. ${ }^{4}$ The clinical presentation of PPCM differs widely, and the rates of recovery of LV function also differ from $23 \%$ to $66 \%$ with regard to study size, race, and follow-up duration. ${ }^{4}$ To date, most of the studies that pertain to PPCM have been performed in the USA, Haiti, and South Africa, and the clinical data are much more limited in the rest of the world. ${ }^{6}$ Although the pathophysiology of PPCM is still not fully understood, advances have been achieved in understanding some underlying molecular circuits in PPCM, which point to multiple causal factors. In particular, excessive oxidative stress and the subsequent cleavage of the nursing hormone prolactin into an antiangiogenic $16 \mathrm{kDa}$ subfragment have emerged as potential causal factors for PPCM. ${ }^{7}$

It is believed that $\mathrm{LV}$ function recovers in $23 \%-41 \%$ of PPCM patients over time, but the literature on right ventricular (RV) systolic dysfunction (RVSD) in PPCM is still limited, and analysis of the RV as well as its systolic function appears in very few studies worldwide. ${ }^{8}$ Currently, the study of the RV is relevant, particularly because of its prognostic's interest in several heart diseases. Moreover, the validation of several echocardiographic indexes allows a better analysis of its overall function. ${ }^{9}$ The aim of this study was to assess the prevalence of RV dysfunction in the PPCM population using longitudinal indexes and new indexes.

\section{Methods}

This is a prospective and observational study performed at the Cardiac Clinic of the Aristide Le Dantec Teaching Hospital in Dakar, Senegal, between March and June 2013. We consecutively recruited all who were hospitalized in the department for a recent diagnosis of PPCM with Doppler echocardiography.

The studied parameters were demographic (age, race, and socioeconomic level), clinical (blood pressure, heart rate, and signs of cardiac failure), biological (blood count, sedimentation rate, blood ionogram, 24-hour proteinuria, and uric acid), electrocardiographic, and echocardiographic variables. The cardiac Doppler echocardiography was carried out by a consultant cardiologist who was trained in echocardiography, with $>10$ years of experience in this field, following the recommendations of the American Society of Echocardiography. ${ }^{9}$ The dimensions of the RV (diameter and pumping chamber) and the surface of the right atrium were measured on several sections. ${ }^{9}$

The systolic function was studied using several parameters such as the tricuspid annular plane systolic excursion (TAPSE).
We have also combined other indexes for better sensitivity and specificity, in particular, the speed of the systolic wave to the tricuspid ring in tissue Doppler (tricuspid Sa wave), the peak of isovolumetric contraction rate (IC Vmax); myocardial acceleration and eccentricity index of the RV (computed by the telesystole and telediastole ratio of the anteroposterior and septal to lateral diameters of the LV in the medioventricular axis). Isovolumetric myocardial acceleration (IVA), RV surface shortening, the ratio between the sum of both isovolumic times and the ejection time (TEI index), as measured in this pulsed Doppler study, were also investigated.

The standards for the RV systolic function indexes were those of the American Society of Echocardiography: ${ }^{9}$ TAPSE $\geq 16 \mathrm{~mm}$, tricuspid wave $\geq 10 \mathrm{~cm} / \mathrm{m}$, IC Vmax $\geq 13 \mathrm{~m} / \mathrm{s}$, IVA $\geq 2.2 \mathrm{~m} / \mathrm{s}^{2}$, fractional area change (FAC) $\geq 35 \%$, and TEI index $0.15-0.40$.

In the absence of magnetic resonance imaging (MRI) as a baseline test for RV systolic function, we considered that there was a RVSD when there were 3 positive indexes that should necessarily contain one of the following indexes: FAC, TAPSE, Sa tricuspid wave (speed of the systolic wave to the tricuspid ring in tissue Doppler), or the TEI index.

The study of diastolic function involved the analysis of the tricuspid flood. Thus we measured the wave A, the wave $\mathrm{E}$, the $\mathrm{E} / \mathrm{A}$ ratio, and the deceleration time of the tricuspid wave $\mathrm{E}$. The tricuspid wave was also studied to calculate the E/e' ratio. The lower limit for the tricuspid wave was $8 \mathrm{~cm} / \mathrm{s}$. Finally, we measured the isovolumetric relaxation time of the RV (IRTRV) in tissue Doppler.

The diastolic function was assessed as follows: an E/A ratio $<0.8$ suggests a relaxation disorder, $0.8<\mathrm{E} / \mathrm{A}<2.1$ with an $\mathrm{E} / \mathrm{e}$ ' ratio $>6$ suggests a pseudonormal aspect, and an $\mathrm{E} / \mathrm{A}$ ratio $>2.1$ with a deceleration time of the wave $\mathrm{E}<120 \mathrm{~ms}$ suggests restrictive flow.

We evaluated pulmonary pressures by analysis of tricuspid insufficiency flux and/or pulmonary insufficiency flux. We also studied other parameters that may be relevant to the indirect diagnosis of pulmonary hypertension (PHT), which is the pulmonary acceleration time as well as the time of isovolumetric relaxation of the RV. A systematic search for the pulmonary mesosystolic notch was also performed.

An IRTRV in tissue Doppler of $<40 \mathrm{~ms}$ would rule out presence of $100 \%$ PHT, while its elongation would indicate either PHT or a dysfunction of this ventricle. The pulmonary mesosystolic notch is suggestive of PHT.

The data were entered and analyzed on Epi Info v6.0. The continuous variables were expressed by the mean \pm SD. Student's $t$-test and the chi-square test were performed for 
comparisons between groups when these were appropriate, and when the sample was too small or there was abnormal distribution we used the Mann-Whitney $U$-test or Fisher's exact test. A $p$-value of $<0.05$ was regarded to be statistically significant.

\section{Ethics approval and consent to participate}

The study protocol was approved by the ethics committee of the Department of Cardiology, Aristide Le Dantec Teaching Hospital. All the patients recruited signed an informed consent form to participate in the study. Those included were all patients with PPCM who met the criteria defined by the Task Force of the European Society of Cardiology in $2008 .^{1}$

\section{Results}

\section{General characteristics of the study population}

During the study period, 326 patients were hospitalized in the Department of Cardiology, and 19 were diagnosed with PPCM, with a hospital prevalence of $5.8 \%$ for PPCM.

Table 1 shows the general characteristics of all patients with or without systolic dysfunction of the RV.

\section{RV systolic function}

Eleven patients $(57.89 \%)$ had dilatation of RV and right atrium, with an average area of the left atrium of $20.06 \pm 6.61 \mathrm{~cm}^{2}$ (Table 2). Fifteen patients (78.9\%) had at least 3 of the following indexes (FAC, Sa tricuspid wave, TAPSE, and TEI index) altered (Table 3). We present the values of the various indexes used to assess the RV systolic function FAC (Figure 1A and B), TAPSE and Sa T (Figure 2A and B), and TEI index (Figure 3).

\section{RV diastolic function}

The average E/A ratio was $1.34 \pm 0.24$, with extremes of 0.78 and 1.6.

There was only 1 case of relaxation disorder, with an E/A ratio of 0.78 and no restrictive flux. Eight patients (42.1\%) had an $\mathrm{E} / \mathrm{e}^{\prime}$ ratio above the norm. All patients had a high IRTRV, with an average of $62.68 \pm 36.52 \mathrm{~ms}$ and extremes of $62.68 \pm 36.52 \mathrm{~ms}$

Overall, we found 1 case of RV relaxation disorder $(5.26 \%), 8$ cases of pseudonormal profile (42.1\%), and no restrictive profile. Of these patients, 6 had their right atria dilated and all had an elongated IRTRV.
Table I Overview of the study population

\begin{tabular}{|c|c|c|}
\hline Characteristics & $\begin{array}{l}\text { PPCM } \\
\text { population } \\
(n=19)\end{array}$ & Percentage \\
\hline Age, years & $31.7 \pm 6.9$ & \\
\hline \multicolumn{3}{|l|}{ Number of pregnancies } \\
\hline$\leq 2$ & 9 & 47.4 \\
\hline$>2$ & 10 & 52.6 \\
\hline \multicolumn{3}{|l|}{ Number of deliveries } \\
\hline$\leq 2$ & 9 & 47.4 \\
\hline$>2$ & 10 & 52.6 \\
\hline Twin pregnancy & 0 & - \\
\hline \multicolumn{3}{|l|}{ Socioeconomic level } \\
\hline Low & 15 & 78.9 \\
\hline Medium & 4 & 21.1 \\
\hline \multicolumn{3}{|c|}{$\begin{array}{l}\text { Time between appearance of symptoms } \\
\text { and delivery }\end{array}$} \\
\hline$\leq 4$ weeks & 12 & 63 \\
\hline 4-12 weeks & 4 & 21 \\
\hline$>12$ weeks & 3 & 16 \\
\hline \multicolumn{3}{|l|}{ NYHA } \\
\hline Class 2 & - & - \\
\hline Class 3 & 13 & 68.4 \\
\hline Class 4 & 6 & 31.6 \\
\hline Median SBP, mmHg & $114.2 \pm 20.34$ & - \\
\hline Median DBP, mmHg & $81.05 \pm 14.83$ & - \\
\hline Hypertension & 4 & 21 \\
\hline Heart rate & $113 \pm 9.26$ & - \\
\hline Right-sided heart failure & 16 & 84.2 \\
\hline Hemoglobin level, g/dL & $11.92 \pm 3.44$ & - \\
\hline LVH (EKG) & 2 & 10.5 \\
\hline HVD (EKG) & 8 & 42.1 \\
\hline Arrhythmia & I & - \\
\hline Mean LVED, mm & $58.42 \pm 5.73$ & - \\
\hline Mean LVES, mm & $51.78 \pm 4.91$ & - \\
\hline Mean LVEF (Simpson biplane) & $23.01 \pm 9.73$ & - \\
\hline
\end{tabular}

Note: All the values are expressed as average \pm standard deviation or number and percentage.

Abbreviations: DBP, diastolic blood pressure; EKG, electrocardiogram; LVED, left ventricle end-diastolic diameter; LVEF, left ventricular ejection fraction; LVES, left ventricle end-systolic diameter; LVH, left ventricular hypertrophy; NYHA, New York Heart Association; PPCM, peripartum cardiomyopathy; SBP, systolic blood pressure.

Table 2 Dimensions of right ventricle

\begin{tabular}{ll}
\hline Dimensions & Average \pm standard deviation \\
\hline Right ventricle TM mode & $26.94 \pm 5.61 \mathrm{~mm}$ \\
Right ventricle basal & $43.05 \pm 7.84 \mathrm{~mm}$ \\
Right ventricle median & $35.36 \pm 8.92 \mathrm{~mm}$ \\
Proximal diameter & $32.36 \pm 5.97 \mathrm{~mm}$ \\
Distal diameter & $31.2 \pm 4.15 \mathrm{~mm}$ \\
Area of right atrium & $20.06 \pm 6.61 \mathrm{~cm}^{2}$ \\
\hline
\end{tabular}

Abbreviation: TM, time-motion.

\section{Pulmonary pressures}

In total, after assessing the results of systolic pulmonary arterial pressure (PAPs) by age, we found 13 cases $(68.4 \%)$ of PHT, with an average PAPs value of $58.46 \mathrm{mmHg}$. 
The mean pulmonary acceleration time was $85.33 \pm$ $29.33 \mathrm{~ms}$, with extremes of 55 and $163 \mathrm{~ms}$. Two patients had a pulmonary acceleration time $>110 \mathrm{~ms}$, with PAP values of 29 and $21 \mathrm{mmHg}$.

Four patients had a mesosystolic notch on their pulmonary flow, with a short pulmonary acceleration time of $69 \mathrm{~mm}$

Table 3 Evaluation of right ventricle systolic dysfunction by the different indexes

\begin{tabular}{lll}
\hline Index & $\begin{array}{l}\text { Average } \pm \text { standard } \\
\text { deviation }\end{array}$ & $\begin{array}{l}\text { Prevalence of RV } \\
\text { systolic dysfunction } \\
\text { by index }(\mathbf{n}=19)\end{array}$ \\
\hline LV-EI & & $8(42.1 \%)$ \\
$\quad$ End-systolic & $1.01 \pm 0.12$ & - \\
$\quad$ End-diastolic & $1.02 \pm 0.14$ & - \\
TAPSE, mm & $14.73 \pm 3.89$ & $1 \mathrm{I}(57.9 \%)$ \\
TASV, cm/s & $10.05 \pm 2.98$ & $1 \mathrm{I}(57.9 \%)$ \\
IVV, m/s & $11.12 \pm 5.3$ & $1 \mathrm{I}(57.9 \%)$ \\
IVA, m/s & $2.83 \pm 1.12 \mathrm{~m} / \mathrm{s}^{2}$ & $7(36.84 \%)$ \\
Mean TEl index & $0.72 \pm 0.63$ & $13(68.4 \%)$ \\
RVFAC & $23.73 \pm 14.16 \%$ & $15(78.9 \%)$
\end{tabular}

Abbreviations: IVA, myocardial acceleration during isovolumetric contraction; IVV, myocardial velocity during isovolumetric contraction; LV-El, left ventricle eccentricity index; RV, right ventricle; RVFAC, right ventricular fractional area change; TAPSE, tricuspid annular plane systolic excursion; TASV, tricuspid annular systolic velocity; TEl, ratio between the sum of both isovolumic times and the ejection time. due to pulmonary hypertension (Figure 4); all had PHT, of which, 2 were severe.

\section{Evolution}

Complications during hospitalization were shock (1 case), requiring initiation of a positive inotropic drug with an improvement in hemodynamic status, and 1 case of intracavitary thrombus that later became complicated. No deaths were recorded during the study period.

\section{Discussion}

All 19 patients with PPCM had LV dysfunction, with an average ejection fraction of $23.01 \% \pm 9.73 \%$, calculated at the Simpson biplane.

Based on the TAPSE, 11 out of 19 patients $(57.9 \%)$ had RVSD. When the other parameters were taken into account, 15 patients $(78.9 \%)$ had at least 3 of the following indexes altered: FAC, Sa tricuspid, TAPSE, and TEI index. A study of RVSD in patients with PPCM in Nigeria found a prevalence of $54.6 \%$ according to TAPSE alone and this prevalence increased to $71.1 \%$ when combined with Sa tricuspid. ${ }^{10}$ Based on the RV fractional area, we found $73.6 \%$ patients with RV
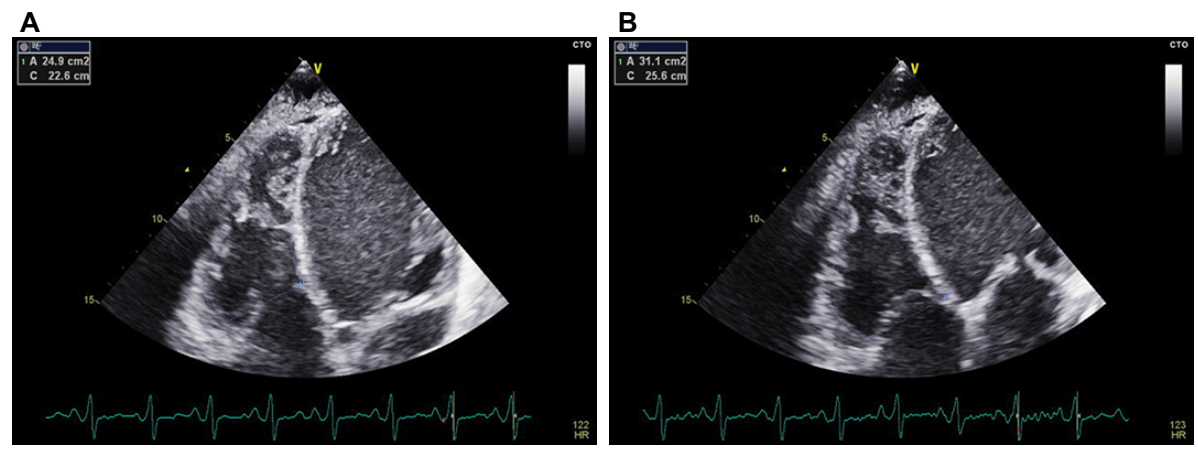

Figure I RV dysfunction with RV shortening fraction of $19.3 \%$ in a patient with PPCM.

Notes: Measurement of fractional linear shortening obtained by measuring the RV diameter at end-diastole (A) and end-systole (B) using the apical view. Abbreviations: PPCM, peripartum cardiomyopathy; RV, right ventricle.
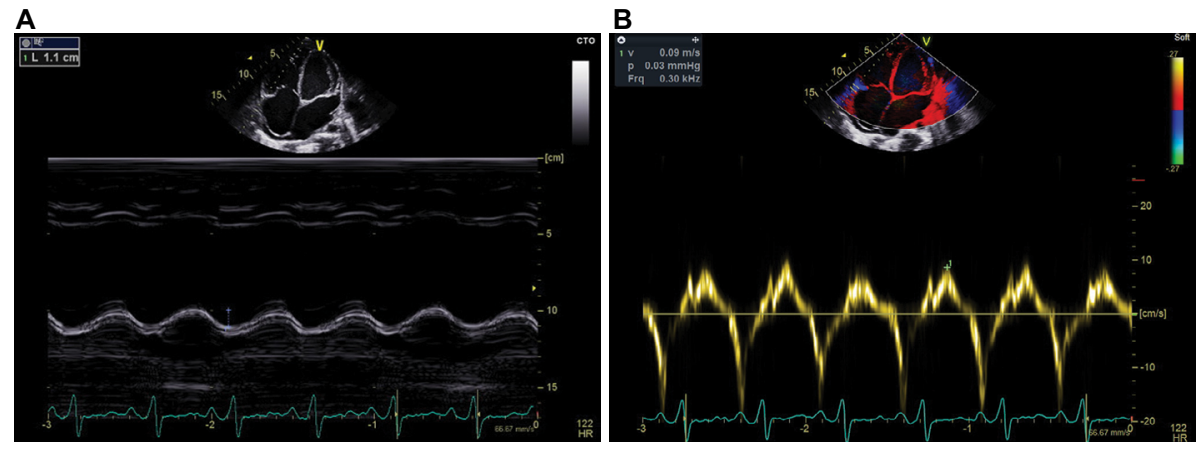

Figure 2 RV dysfunction with TAPSE $=11 \mathrm{~mm}$ and $\mathrm{Sa} T=9 \mathrm{~cm} / \mathrm{s}$.

Notes: (A) Tricuspid annular plane systolic excursion (TAPSE) measured at the lateral tricuspid annulus in TM Mode. (B) Myocardial systolic excursion velocity (Sa tricuspid) displayed with DTI using the apical four cavity view at the lateral.

Abbreviations: DTI: Doppler tissue imaging; RV, right ventricle; Sa T, speed of the systolic wave to the tricuspid ring in tissue Doppler; TAPSE, tricuspid annular plane systolic excursion; TM, time-motion. 


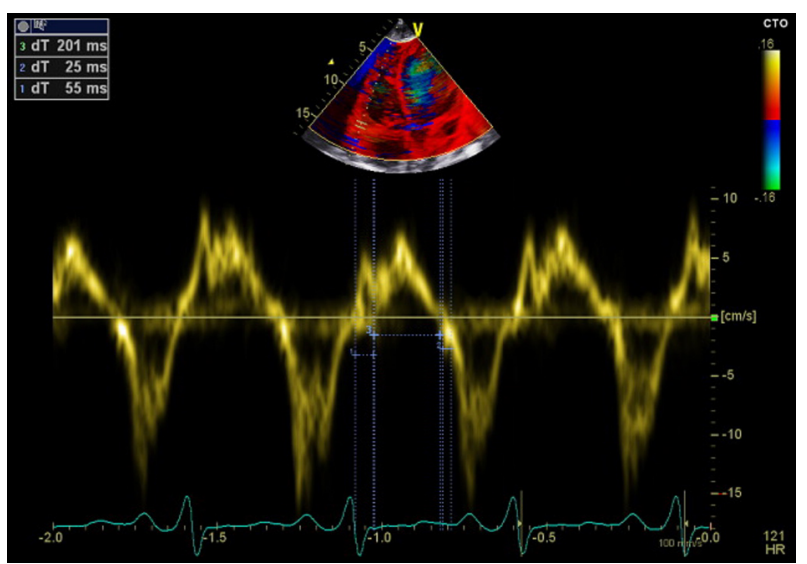

Figure 3 TEl index $=0.60$.

Abbreviation: TEI, the ratio between the sum of both isovolumic times and the ejection time.

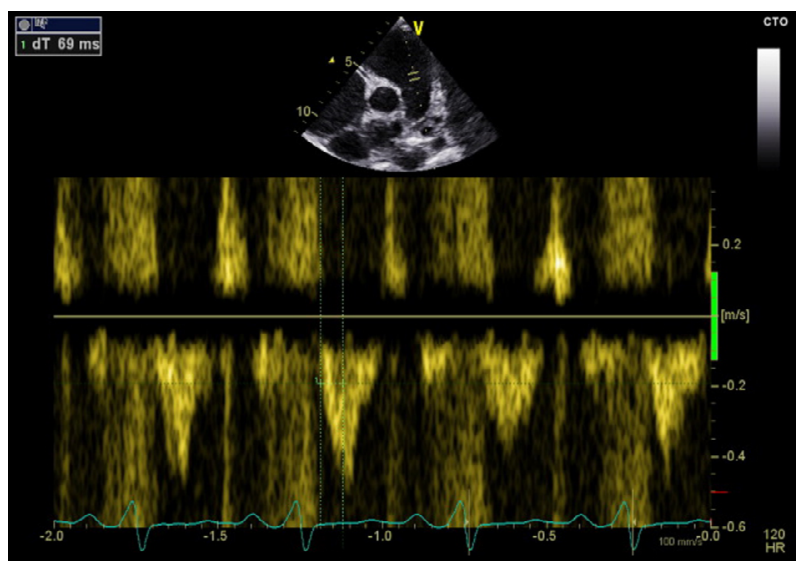

Figure 4 Short pulmonary acceleration time at $69 \mathrm{~ms}$ due to pulmonary hypertension.

dysfunction versus $88.4 \%$ in the literature. ${ }^{8}$ Similar to a study by Karaye, ${ }^{7}$ PHT was found in $68.4 \%$.

The precise mechanisms that lead to PPCM remain poorly defined. Many pathogenetic conditions have been suggested: viral myocarditis, an abnormal immune response to fetal microchimerism (harboring of fetal cells in maternal circulation), a maladaptive response to the hemodynamic stresses of pregnancy, stress-activated cytokines, and prolonged tocolysis. ${ }^{12}$ Furthermore, a familial predisposition to PPCM has been reported. ${ }^{12}$ In addition, nutritional disorders, such as deficiencies in selenium and other micronutrients, might play a role in the pathogenesis of PPCM. ${ }^{12}$ Karaye et $\mathrm{al}^{12}$ described, in a cohort of 43 subjects with PPCM, that a high proportion of selenium deficiency and combined RVSD and PHT seemed to be the only determinants of RVDD. As the $\mathrm{RV}$ dysfunction is discovered at the same time as the LV dysfunction, the question arises whether there is a different distinct pattern of LV wall motion abnormalities. Whether specific pathomechanisms are responsible for RV disease involvement in PPCM require further investigation.

Besides the frequency of RV involvement, we note that the clinical profile of the patients in this work is similar to that already reported in the literature, notably as regards the etiological factors such as the age of occurrence around $31.7 \pm 6.9$ years, multiparity, prevalence of twins, and low socioeconomic status. ${ }^{2,11-14}$ In addition, there were reports of signs of RV failure, ${ }^{4,5,11}$ which were noted in $84 \%$ of the patients in this work.

Because RV dysfunction in PPCM emerged as a negative predictor for heart disease outcome, clinicians should also focus on RV dysfunction in PPCM patients at first diagnosis and in follow-up analyses, as it might affect patient morbidity and may therefore have implications on therapy, risk stratification, and management. ${ }^{7}$ Since we do not use MRI in common practice for the diagnosis of RV involvement in PPCM, we emphasize in this study the importance of using several of these effects and complementary parameters for better sensitivity and specificity in the evaluation of the RV. Cardiac Doppler echocardiography is an essential contribution to the diagnosis of RV attacks; its accessibility and reproducibility makes it an examination of choice under our conditions, wherein the MRI is more difficult to obtain due to the high cost or its nonavailability in all centers.

This study certainly has some limitations, such as the low number of patients in the study and the absence of MRI for the confirmation of RV involvement since it is known that it allows a better study of the ventricular involvement; ${ }^{7}$ but, echocardiography remains an accessible, reproducible, and less-expensive tool in the context of our country being a developing nation. We will also include among the limits the inability to perform coronary artery angiography to exclude with certainty an ischemic attack in our patients.

\section{Conclusion}

This study evaluated the prevalence of systolic and diastolic involvement of the RV and pulmonary arterial hypertension in a group of 19 patients with PPCM who live in Dakar, Senegal. This prevalence is better evaluated by the use of several combined parameters in the absence of MRI. This study is the first that combines the evaluation of RV function by several valid parameters based on the latest recommendations of the American Society of Echocardiography. The analysis of the RV, as well as its systolic function, appear only in a few studies on PPCM, and RV dilatation and dysfunction were common in this population. 


\section{Acknowledgments}

We acknowledge Dioum M (Department of Cardiology, Teaching Hospital of Fann, Dakar, Senegal), Mingou J, Gaye ND, and Kana SB (Department of Cardiology, Aristide Le Dantec Teaching Hospital, Dakar, Senegal), Leye M (Department of Cardiology, Teaching Hospital of Fann, Dakar, Senegal), and Ndao CT (Department of Cardiology, Aristide Le Dantec Teaching Hospital, Dakar, Senegal) for substantial contributions to this manuscript.

\section{Author contributions}

MBN, SAS, and FA conducted the patients' echocardiography analysis and interpreted the patient data; FA wrote this manuscript; AAN, collected the data, analyzed and interpreted the data; AM, Adama K, Abdoul K, and SAB contributed to the correction of this manuscript. All authors contributed toward data analysis, drafting and critically revising the paper and agree to be accountable for all aspects of the work. All authors read and approved the final manuscript.

\section{Disclosure}

The authors report no conflicts of interest in this work.

\section{References}

1. Elliott P, Andersson B, Arbustini E, et al. Classification of the cardiomyopathies: a position statement from the European Society of Cardiology working group on myocardial and pericardial diseases. Eur Heart J. 2008;29(2):270-276.

2. Kane A, Dia AA, Diouf A, et al. Myocardiopathie idiopathique du péripartum: étude prospective échocardiographique. Ann Cardiol Angeiol. 2001;50:305-311.
3. Bouabdallaoui N, Mouquet F, Lebreton G, Demondion P, Le Jemtel TH, Ennezat PV. Current knowledge and recent development on management of peripartum cardiomyopathy. Eur Heart J Acute Cardiovasc Care. 2015;6(4):359-366.

4. Sliwa K, Hilfiker-Kleiner D, Petrie MC, et al; Heart Failure Association of the European Society of Cardiology Working Group on Peripartum Cardiomyopathy. Current state of knowledge on aetiology, diagnosis, management, and therapy of peripartum cardiomyopathy: a position statement from the heart failure association of the European Society of Cardiology working group on peripartum cardiomyopathy. Eur J Heart Fail. 2010;12(8):767-778.

5. Li W, Li H, Long Y. Clinical characteristics and long-term predictors of persistent left ventricular systolic dysfunction in peripartum cardiomyopathy. Can J Cardiol. 2016;32(3):362-368.

6. Haghikia A, Röntgen P, Vogel-Claussen J, et al. Prognostic implication of right ventricular involvement in peripartum cardiomyopathy: a cardiovascular magnetic resonance study. ESC Heart Fail. 2015;2(4):139-149.

7. Karaye KM, Lindmark K, Henein M. Right ventricular systolic dysfunction and remodelling in Nigerians with peripartum cardiomyopathy: a longitudinal study. BMC Cardiovasc Disord. 2016;16:27.

8. Blecker GB, Steendijk P, Holman ER, et al. Assessing right ventricular function: the role of echocardiography and complementary technologies. Heart. 2006;92(suppl 1):i19-i26.

9. Rudski LG, Lai WW, Afilalo J, et al. Guidelines for the echocardiographic assessment of the right heart in adults: a report from the American Society of Echocardiography endorsed by the European Association of Echocardiography, a registered branch of the European Society of Cardiology, and the Canadian Society of Echocardiography. JAm Soc Echocardiogr. 2010;23(7):685-713.

10. Karaye KM. Right ventricular systolic function in peripartum and dilated cardiomyopathies. Eur J Echocardiogr. 2011;12(5):372-374.

11. Scardovi AB, De Maria R. Peripartum cardiomyopathy: challenges and solutions. Res Rep Clin Cardiol. 2017;2017(8):31-40.

12. Karaye KM, Lindmark K, Henein M. Prevalence and predictors of right ventricular diastolic dysfunction in peripartum cardiomyopathy. $J$ Echocardiogr. Epub Feb 28, 2017.

13. Sliwa K, Fett J, Elkayam U. Peripartum cardiomyopathy. Lancet. 2006;368(9536):687-693

14. Akil MA, Bilik MZ, Yildiz A, et al. Peripartum cardiomyopathy in Turkey: experience of three tertiary centres. J Obstet Gynaecol. 2016;36(5): 574-580.
Research Reports in Clinical Cardiology

\section{Publish your work in this journal}

Research Reports in Clinical Cardiology is an international, peerreviewed, open access journal publishing original research, reports, editorials, reviews and commentaries on all areas of cardiology in the clinic and laboratory. The manuscript management system is completely online and includes a very quick and fair peer-review system.

\section{Dovepress}

Visit http://www.dovepress.com/testimonials.php to read real quotes from published authors. 